\title{
Höchste Verbundfestigkeit gezeigt
}

Eine aktuelle In-vitro-Untersuchung an der Universität Regensburg zur Verbundfestigkeit vorgefertigter Verblendschalen weist auf einen anhaltenden Haftverbund

der Schalen von Heraeus Kulzer hin. Sie bestätigt höchste Verbundfestigkeit von PalaVeneer in Kombination mit dem zugehörigen Verbundsystem, dem kaltpolymerisierenden
PMMA-Kunststoff PalaVeneer Dentine, zum Metallgerüst. Der Einsatz der neuen PalaVeneer-Verblendschalen bietet sich gerade bei geringen Platzverhältnissen an, z. B. bei Doppelkronenarbeiten, Teleskoparbeiten oder implantatgetragenen Konstruktionen. Hier können Zahntechniker zeitaufwendiges Ausschleifen von Vollzähnen umgehen. Detaillierte Informationen zu der wissenschaftlichen Studie finden Sie hier: heraeus-kulzer.de/PalaVeneer_Studie

Nach einer Pressemitteilung der Heraeus Kulzer $\mathrm{GmbH}$, Hanau www.heraeus-kulzer.de 